\title{
Research on Node Deployment in Structural Health Monitoring Based on Reliability
}

\section{Zhenya Zhu ${ }^{1}$}

East East China University Science and Technology

Shanghai, 200000, China

E-mail: hongyizhuge@163.com

\section{Fangming Shao ${ }^{2}$}

East East China University Science and Technology

Shanghai, 200000, China

E-mail: fmshaodecust.com.cn

\section{Zuyuan Zhang ${ }^{3}$}

East East China University Science and Technology

Shanghai, 200000, China

E-mail: yuan819170178@126.com

\begin{abstract}
Structural Health Monitoring (SHM) systems are used to monitor the operation and health status of large infrastructures. As wireless sensors require low power and are easily deployable, the Wireless Sensor Networks (WSN) are more commonly in the applications of applied to the system than the wired networks. However, SHM brings new challenges to WSNs such as engineering-driven optimal deployment, a large volume of data, sophisticated computing, etc., and so forth. In order to optimize the deployment of wireless sensors in the system, we introduce the reliability method to evaluate the network performance and consider an optimization problem: how to deploy the fewest backup sensor nodes such that the reliability is not no less than a given value $R_{0}$ after the primary locations are have been determined. As a result, we prove that the reliability of $G^{\prime}$ (the added node is linked to every node in $G$ ) has been proved is higher than that of $G$. Accordingly, we propose an optimization optimized strategy of backup sensors. The simulation shows the effectiveness of the proposed strategy.
\end{abstract}

CENet 2017

22-23 July, 2017

Shanghai, China

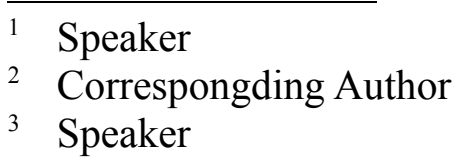




\section{Introduction}

The Structural Health Monitoring (SHM) is the dynamic response of sensors and collects information of changes over time to estimate the system's sensitivity to the structural damage. The health of the structure is reflected by the collected data, such as security, building materials damage and deformation limit. The current SHM is mainly implemented by manpower or wired sensors, which are inefficient and unable to lasting for long time. Due to the low power and easy deployment of wireless sensors, the Wireless Sensor Networks (WSN) are becominghas become an enabling technology for SHM applications. Moreover, the new progress about sensor technology makes WSN effective and generates economical solution for a variety of applications, such as target tracking, scientific exploration and environmental monitoring [1-3], multi-sensor intelligent development has have been the trend of SHM system.

Many researches focused on the assessment models to estimate the remaining useful life (RUL) $[4,5]$ and health performance [6,7] in the engineering domain. In order to decrease the losses caused by resulted from the large-scale breakdown in interdependent network [8], infrastructure networks [9] and complex dynamical network [10], they presented cascading failure analysis and restoration strategy. SHM systems monitor the health status of the structure and provide long-term monitoring and rapid analysis of unusual events such as earthquakes, loads, etc. In practice, it is difficult to achieve the goal because of the requirements of on SHM and the severe limitations of WSNs [1,3]. One of the basic requirements for on SHM is the sensor location optimization. In the civil engineering, wireless sensors are usually deployed at strategic locations to achieve optimal estimates of structural health [11,12]. These methods do not support the sensor deployment at anytime or anywhere in the structure. They firstly focused on the deployment of a set of wireless sensors (called primary sensors), effective independence (EFI) $[11,13,14]$. Each location in the structure correspondss to an EFI value, which is an effective way to determine the primary locations of the structure.

For SHM, recent researches [3,15] optimized the deployment of wireless sensors based on EFI, which is an indication of the performance of deployment. The larger the value was, the better the placement would be. Bhuiyan et al. [16] proposed that the SHM systems need demanded fault tolerance based on WSNs, subject to network still operation when some faults occur in the WSN. They proposed BSP algorithm to find the locations of backup sensors to improve the fault tolerance of network.

We consider the strategy of sensor placement based on the node failure reliability model. In WSN, we denote the communication radius of sensor as $r_{s}$. If the distance between two sensors is less than $r_{s}$, an edge is generated between the two sensors. As to the reliability, we introduce Reliability on node-failure(RNF) [17]: Suppose suppose the edges of graph $G$ never fail and the nodes fail independently with probability $1-p$. Then the reliability of graph $G$ is

$$
R(G, p)=\sum_{r=1}^{n} S_{r}(G) p^{r}(1-p)^{n-r}
$$

where $S_{r}(G)$ is the number of connected induced subgraphs of graph $G$ that contains exactly $r$ nodes.

\section{System Model and Problem Formulation}




\subsection{System Model}

We assume that there are $M$ candidate locations and $N_{1}$ primary sensors in a structure. Generally, $N_{1}$ primary sensors are deployed in $M$ candidate locations according to EFI value. In the SHM system, the locations state and sensor quantity can be stated mathematically as:

$$
X=\left[\begin{array}{cccc}
x_{11} & x_{12} & \ldots & x_{1 m} \\
x_{21} & x_{12} & \ldots & x_{1 m} \\
\ldots & \ldots & \ldots & \ldots \\
x_{n 1} & x_{n 2} & \ldots & x_{n m}
\end{array}\right] \quad \Lambda=\left[\begin{array}{cccc}
\lambda_{11} & \lambda_{12} & \ldots & \lambda_{1 m} \\
\lambda_{11} & \lambda_{22} & \ldots & \lambda_{2 m} \\
\ldots & \ldots & \ldots & \ldots \\
\lambda_{n 1} & \lambda_{n 2} & \ldots & \lambda_{n m}
\end{array}\right]
$$

where $X$ is refers to the structure position matrix, $x_{i j}(1 \leq i \leq n, 1 \leq j \leq m)$ is refers to the state variable of the location $(i, j): x_{i j}=0$ when the location $(i, j)$ is empty; $x_{i j}=1$ when the location $(i, j)$ is not empty. $\Lambda$ is the sensor quantity matrix, $\lambda_{i j}\left(0 \leq \lambda_{i j} \leq 2\right)$ is the number of sensors deployed in location $(i, j)$.

Definition 1. The number of non-empty locations is:

$$
S(X)=\sum_{i}^{n} \sum_{j}^{m} x_{i, j}
$$

Let $X^{0}$ be the position matrix after deploying primary sensors, then the number of primary locations is $N_{1}=S\left(X^{0}\right)=\sum_{i}^{n} \sum_{j}^{m} x_{i, j}^{0} \quad$ from Definition 1. The number of new deployed locations is $B(X)=S(X)-S\left(X^{0}\right)$, and $B(X) \in\left(0, M-N_{1}\right)$. From (2.1), when one location can be deployed multiple sensors, the number of new deployed sensors $B(\Lambda)$ is

$$
B(\Lambda)=S(\Lambda)-S\left(E X^{0}\right)=\sum_{i}^{n} \sum_{j}^{m} \lambda_{i, j}-\sum_{i}^{n} \sum_{j}^{m} x_{i, j}
$$

where $S(\Lambda)$ is the number of sensors in $\Lambda$.

Definition 2. $f(\Lambda)$ represents a function of the reliability of $G$ with sensor quantity matrix $\Lambda$, and

$$
f(\Lambda)=R(G)
$$

For the deployment problem, converting location topology to WSN topology while preserving its reliability is of practical importance. By mathematical descriptions and definitions, the deployment problem and the reliability optimization can be easy to understand.

\subsection{Mathematical Description of the Problem.}

Given the premise that one backup sensor at most is deployed at each location, we consider an optimization problem of network reliability: to find the fewest backup sensors such so that $R \geq R_{0}$ after the primary sensors have been determined.

For the given candidate locations $(M)$, the primary sensors $\left(N_{1}\right)$ and the backup sensors $\left(N_{2}\right)$ (where $N_{1}+N_{2} \leq M$ ) in SHM, the optimization problem of sensor deployment can be described mathematically as follows:

$\min B(\Lambda)$ 


$$
\begin{array}{ll}
\text { s.t. } & f(\Lambda) \geq R_{0}, \\
& B(\Lambda) \leq N_{2}, \\
& B(X) \leq M-N_{1} .
\end{array}
$$

where the numbers of structure position matrix $X$ and sensor quantity $\Lambda$ states are $2^{m n}$ and $3^{m n}$ at most respectively.

To solve (2.5), the time consumed by the enumeration method grows exponentially with the size of the structure. Then, so we solve the optimization problem by developing new theorems and technique. We append a method to increase the graph reliability after adding a backup sensor.

Theorem 1. The graph $G\left(V^{\prime}, E^{\prime}\right)$ is obtained by adding a node $y$ to $G(V, E)$, if $d(y)=|V|$, then $R\left(G\left(V^{\prime}, E^{\prime}\right), p\right) \geq R(G(V, E), p)$ and $R\left(G\left(V^{\prime}, E^{\prime}\right), p\right)=p+q R(G(V, E), p)$.

Proof: In Figure 1, Let graph $G(V, E)$ as a node, and its reliability is: $R(G(V, E))=\sum_{r=1}^{n} S_{r}(G) p^{r}(1-p)^{n-r}$. Then the reliability of $G\left(V^{\prime}, E^{\prime}\right)$ is:

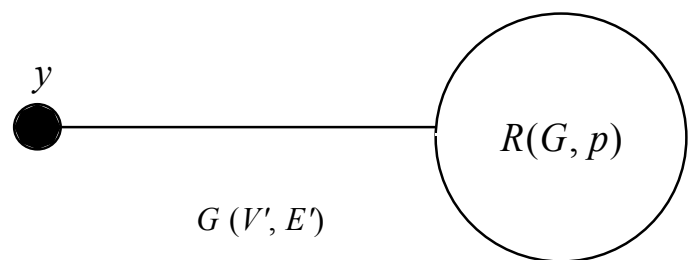

Figure 1: The node y connects with every node in $G(V, E)$

$$
\begin{aligned}
& R\left(G\left(V^{\prime}, E^{\prime}\right), p\right)=p\left(1-\sum_{r=1}^{n} S_{r}(G) p^{r}(1-p)^{n-r}\right)+\sum_{r=1}^{n} S_{r}(G) p^{r}(1-p)^{n-r}(1-p) \\
& +p \sum_{r=1}^{n} S_{r}(G) p^{r}(1-p)^{n-r}=p+(1-p) \sum_{r=1}^{n} S_{r}(G) p^{r}(1-p)^{n-r}=p+q R(G(V, E), p) . \\
& \text { Then } R\left(G\left(V^{\prime}, E^{\prime}\right), p\right)-R(G(V, E), p)=p+(1-p) \sum_{r=1}^{n} S_{r}(G) p^{r}(1-p)^{n-r} \\
& -\sum_{r=1}^{n} S_{r}(G) p^{r}(1-p)^{n-r}=p+q R(G(V, E), p)=p-p R(G(V, E), p)=p(1-R(G(V, E), p)) \geq 0
\end{aligned}
$$

Hence $R\left(G\left(V^{\prime}, E^{\prime}\right), p\right) \geq R(G(V, E), p)$.

The theorem gives a method to increase the reliability of graph $G$ with the added node linked to all other nodes.

\section{Algorithm and Simulation}

According to Theorem 1, we propose an algorithm INR- $R_{0}$ to deploy backup sensors.

Algorithm: INR- $R_{0}\left(M, N_{1}, N_{2}, G, p\right)$
Input: graph $G(V, E)$, total locations $M$, primary sensors $N_{1}$, backup sensors $N_{2}$, the
reliability $R_{0}$, node probability $p$.
Output: Network reliability $R\left(G^{*}\right)$, the number of backup sensors $N_{2}{ }^{*}$.
1:

$$
S=\phi, j=1, R(G)
$$




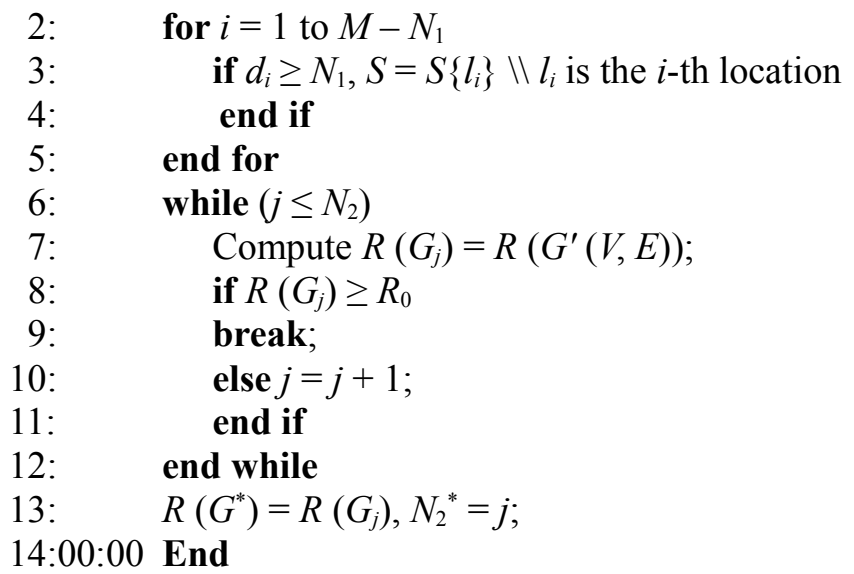

We suppose that each location $l_{i}$ is allowed to deploy one sensor and each step adds one node. INR- $R_{0}$ gives a method to find the location of deploying backup sensors which connectsconnected to all primary locations. Theorem 1 shows that this method can improve the reliability of network. In step 7, we compute $R\left(G_{j}\right)$ by adding $j$ nodes from $S$ to $G$. In addition, the algorithm is executed recursively until $R \geq R_{0}$.

Example 1. The Sensors can be deployed at the dotted line intersection $(M)$. Suppose $v_{1}, v_{2}$, $v_{3}, v_{4}$ are the primary locations $\left(N_{1}\right)$. The number of backup locations are $\left(M-N_{1}\right)$, and that of backup sensor $N_{2}=4$ as shown in Figure 2 shows. The failure probability of each node is $1-p$ $=0.1$. For the given $R_{0}=0.999$ (and the communication radius $r_{s}=3$ ), the results are shown by in Table 1.
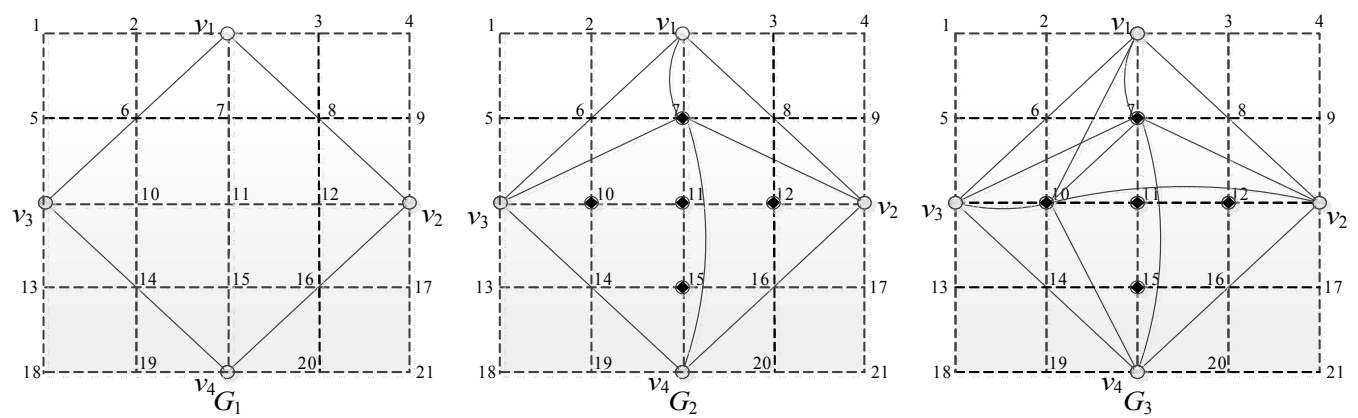

Figure 2: $G_{1}$ :Network with 4 primary sensors, $G_{2}$ :added one backup sensor (black) by INR- $R_{0}$, $G_{3}$ :added two backup sensors

\begin{tabular}{|c|c|c|c|}
\hline Add node & Graph- $G$ & Reliability- $R$ & Compare with- $R_{0}$ \\
\hline Null & $G_{1}$ & 0.9837 & $R<R_{0}$ \\
\hline 7 & $G_{2}$ & 0.99837 & $R<R_{0}$ \\
\hline 10 & $G_{3}$ & 0.999837 & $R>R_{0}$ \\
\hline
\end{tabular}

Table 1:The reliability of graph with adding nodes

Table 1 shows the reliability of graph with added nodes, the reliability of graph $G_{1}$ is $R$ $\left(G_{1}, p\right)=4 p q^{3}+4 p^{2} q^{2}+4 p^{3} q+p^{4}=0.9837<R_{0}$. According to algorithm INR- $R_{0}$ and the communication radius $r \leq 3$, we find the set $S=\{7,10,11,12,15\}$. Firstly, add node 7 in $G_{1}$ to get $G_{2}$, then the reliability of $G_{2}$ is $R\left(G_{2}, p\right)=p+q R\left(G_{1}, p\right)=0.9+0.1 \times 0.9837=0.99837<$ $R_{0}$. As $G_{3}$ shows, add node 10 in $G_{2}$, then the reliability of $G_{3}$ is $R\left(G_{3}, p\right)=p+q R\left(G_{2}, p\right)=0.9$ $+0.1 \times 0.99837=0.999837>R_{0}$. In under this situation, the simulation results are consistent with our theoretical results. 
In Example 1, the strategy that added node connectts to all other nodes can improve the reliability of network.

\section{Conclusion}

In this paper, our goal is to give a way of incorporating the requirements of both WSN and SHM. We found that it is worthwhile to deploy some backup sensors in the WSN to have a better performance. The optimization strategy of sensors deployment in SHM can be described by reliability theory. To solve the optimization problem, we've proved that adding a node which that connects to each node could improves the reliability of network. Accordingly, we present an algorithm to optimize sensor node deployment, test an example and shows the correctness of reliability methods. The future work will focus on the robust of optimal problem.

\section{References}

[1] X. Mao, X. Miao, Y. He, T. Zhu, J. Wang, W. Dong, X. Li, and Y. Liu, "CitySee: Urban CO2 monitoring with sensors," in Proc. IEEE Conf. Comput. Commun. (INFOCOM), 2012, pp. 16111619.

[2] G. Wang, M. Z. A. Bhuiyan, and Z. Li, "Two level cooperative and energy efficient tracking algorithm in wireless sensor networks," Concurrency Comput. Practice and Experience, vol. 22, no. 4, pp518-537, 2010.

[3] M. Z. A. Bhuiyan, G. Wang, J. Cao, and J. Wu, "Energy and bandwidth-efficient wireless sensor networks for monitoring high-frequency events," in Proc. 10th Annu. IEEE Commun. Soc. Conf. Sensor Mesh Ad Hoc Commun. Networks(SECON), 2013.

[4] Hong S, Zhou Z, Zio E, et al. Condition assessment for the performance degradation of bearing based on a combinatorial feature extraction method[J]. Digital Signal Processing, 2014, 27: 159166.

[5] Hong S, Wang B, Li G, et al. Performance Degradation Assessment for Bearing Based on Ensemble Empirical Mode Decomposition and Gaussian Mixture Model[J]. Journal of Vibration \& Acoustics, 2014, 136(6):061006.

[6] Hong S, Zhou Z, Zio E, et al. An adaptive method for health trend prediction of rotating bearings[J]. Digital Signal Processing, 2014, 35: 117-123.

[7] Hong S, Zhou Z, Lu C, et al. Bearing remaining life prediction using Gaussian process regression with composite kernel functions[J]. Journal of Vibroengineering, 2015, 17(2):695-704.

[8] Hong S, Lv C, Zhao T, et al. Cascading failure analysis and restoration strategy in an interdependent network[J]. Journal of Physics A: Mathematical and Theoretical, 2016, 49(19): 195101.

[9] Hong S, Yang H, Zhao T, et al. Epidemic spreading model of complex dynamical network with the heterogeneity of nodes[J]. International Journal of Systems Science, 2016, 47(11): 2745-2752.

[10] Hong S, Wang B, Ma X, et al. Failure cascade in interdependent network with traffic loads [J]. Journal of Physics A: Mathematical and Theoretical, 2015, 48(48): 485101. 
[11] S. Kim, S. Pakzad, D. Culler, J. Demmel, G. Fenves, S, Glaser, and M. Turon, "Health monitoring of civil infrastructures using wireless sensor networks," in Proc. 6th Int. Symp. Inform. Process. Sensor Netw. (IPSN) 2007, pp. 254-263.

[12] G. Hackmann, F. Sun, N. Castaneda, C. Lu, and S. Dyke, “A holistic approach to decentralized structural damage localization using wireless sensor networks," Comput. Commun., Vol. 36, no.1, pp. 29-41, 2012.

[13] K. Chebrolu, B. Raman, N. Mishra, P. K. Valiveti, and R. Kumar, "BriMon: A sensor network system for railway bridge monitoring," in Proc. 6th Int. Conf. Mobile Syst. Appl. Services (MobiSys), 2008, pp. 2-14.

[14] B. Li, D. Wang, F. Wang, and Y. Q. Ni, "High quality sensor placement for SHM systems: Refocusing on application demands," in Proc. IEEE Conf. Comput. Commun. (INFOCOM), 2010, pp. 650-658.

[15] M. Meo and G. Zumpano, "On the optimal sensor placement techniques for a bridge structure," Eng. Struct., vol. 27, no. 2005, pp. 1488-1497, 1991.

[16] M. Z. A. Bhuiyan, J. Cao, G. Wang, and J. Wu, “Deploying wireless sensor networks with faulttolerance for structural health monitoring,” IEEE Trans. Comput., vol. 64, no. 2, pp. 382-395, 2015.

[17] S. Yu, F. M. Shao, and H. Meng, "Uniformly optimal graphs in some classes of graphs with node failures," Discrete Mathematics, vol. 310, no. 1, pp. 159-166, 2010. 